\title{
Intranasal mucoadhesivemicroemulsion for neuroprotective effect of curcuminin mptp induced Parkinson model
}

\author{
Snigdha Das Mandal ${ }^{*}$, Surjyanarayan Mandal², Jayvadan Patel $^{3}$
}

${ }^{1}$ Faculty of Pharmacy, R. K. University, Rajkot, Gujarat, India, ${ }^{2}$ Pharmacy Department, School of Pharmaceutical Sciences, Siksha 'O'Anusandhan University, Khandagiri Square, Bhubaneswar, Orissa, India, ${ }^{3}$ Faculty of Pharmacy, Sankalchand Patel University, Visnagar, Gujarat, India

\begin{abstract}
This study was to investigate the neuroprotective effect of curcumin against inflammation-mediated dopaminergic neurodegeneration in 1-methyl-4-phenyl-1,2,3,6-tetrahydropyridine (MPTP) mice model of Parkinson's disease (PD). Curcumin loaded sodium hyaluronate based mucoadhesive microemulsion (CMME) was developed by using Box Behnken design of Response surface method (RSM) and was characterized. Male C57BL/6 mice were first treated with four intraperitoneal injections of MPTP (20 $\mathrm{mg} / \mathrm{kg}$ of body weight) at $2 \mathrm{~h}$ intervals followed CMME intranasal administration for 14 days at 2.86 $\mathrm{mg}$ of curcumin $/ \mathrm{kg}$ of body weight per once a day. Optimal CMME containing 3\% Capmul MCM as oil phase, $37 \%$ of Accenon CC and Transcutol HP at 2.5:1 ratio and $0.5 \%$ sodium hyaluronate was stable, non-ciliotoxic with $57.66 \mathrm{~nm} \pm 3.46$ as average globule size. PdI value $(0.190 \pm 0.19)$ and TEM result depicted the narrow size distribution of CMME.All three independent variables had a significant effect $(p<0.05)$ on the responses and the designed model was significant for all taken responses. In-vivo results revealed significant reduction of MPTP-mediated dopamine depletion after nasal administration of CMME. MPTP intoxication significantly decreased striatal DA content to $21.29 \%$ which was then elevated to $55.37 \%$ after intranasal curcumin treatment. Significant improvement in motor performance as well as gross behavioural activity of mice was observed from rota-rod and open field test findings. Findings of the investigation revealed the symptomatic neuroprotection of curcumin against MPTP-induced neurodegradation in the striatum and hence could be considered as a promising approach to treat PD.
\end{abstract}

Uniterms: Curcumin/neuroprotective effect. Response surface methodology (RSM). Box-Behnken design. Sodium hyaluronate. Neurodegradation. Parkinson's disease/treatment. Male mice C57BL/6

\section{INTRODUCTION}

Neuroinflammation may contribute to the progressive dopaminergic neuronal loss in substantia nigra pars compacta (SNPS) of brain that has been observed in some experimental models of Parkinson's disease (PD), the second most common neurodegenerative disorder after Alzheimer's disease characterised by a slow and progressive degeneration of dopaminergic neurons in the substantia nigra (Lindvall et al., 2003; Hirsch, Hunot, 2009). Although the etiology of PD remains inscrutable, but some recent advancement reveals the direct involvement of inflammation and oxidation of inflamed substantia nigra pars compacta (SNPS) on

\footnotetext{
*Correspondence: S. D. Mandal. Faculty of Pharmacy. R. K. University. Rajkot, Gujarat, India. E-mail: snigdhadasmandal@gmail.com
}

the PD. PD pathogenesis specifically involved severe dopaminergic neuronal degeneration in the substantia nigra pars compacta ( $\mathrm{SNpc}$ ) and striatum.

In order to understand the pathophysiology of PD, neurotoxin, 1-methyl-4-phenyl-1,2,3,6-tetrahydropyridine (MPTP) which produces parkinsonian syndrome in humans, primates and rodents provides better can be used. MPTP is enzymatically converted into its toxic metabolite $\mathrm{MPP}^{+}$(1-methyl-4-phenylpyridinium) by the enzyme monoamine oxidase-B (MAO-B), primarily in astrocytes of brain and is known to specifically damage dopaminergic neurons in the SNpc (Reznichenko et al., 2010). The neurotoxic effect of $\mathrm{MPP}^{+}$primarily involves the disruption of oxidative phosphorylation and redox homeostasis along with elevation of intra-cellular $\mathrm{Ca}^{2+}$ levels and inflammatory responses (Esposito et al., 2007; Douna et al., 2012). The dopaminergic neuronal 
cell death is due to the inflammatory cascade including microglial activation and excessive secretion of cytotoxic agents like pro-inflammatory cytokines and free radicals. So, considering the importance of inflammation in PD progression, neuroprotective and anti-inflammatory strategies may offer a promising therapeutic intervention for the treatment and management of PD.

Curcumin, the principal curcuminoid of turmeric, has been shown to exhibit antioxidant as well as antiinflammatory. However, the clinical usefulness of curcumin is limited by its poor water solubility and high first-pass metabolism leading to low oral bioavailability and poor brain entry due to over expression of p-glycoprotein (Brown et al., 2012; Miller, Bauer, Hartz, 2008). Therefore, it is worthwhile to develop an alternative but effective approach to improve therapeutic effect of this nontoxic and potent phytoconstituent.

Recently, nasal route becomes one of the most versatile routes of drug administration because it offers several advantages including rapid absorption of drug, avoidance of hepatic first-pass metabolism and more precisely drug delivery preferentially to brain via the olfactory region through nasal turbinates olfactory and trigeminal nerve pathways (Barakat, Omar, Ahmed, 2006). Therefore, the nasal route may provide a better alternative to oral or intravenous administration for the brain delivery of Curcumin.

However, nasal delivery of Curcumin may have several challenges like effective nasal delivery volume and anti-inflammatory dose of Curcumin is very high i.e., 2.86 $\mathrm{mg} / \mathrm{kg}$ of body. Secondly thenatural defense mechanism i.e., nasal mucociliary clearance and metabolism in nasal mucosa (Piaoa et al., 2010). So, formulation like microemulsion may be effective which can improve the solubility of poorly water-soluble Curcumin which can accommodate the effective dose in human i.e., $\leq 400 \mu \mathrm{L}$ (200 $\mu \mathrm{L} /$ nostril). Viscosity of the formulation may play a vital role in prevention of its rapid nasal clearance.

Microemulsions are thermodynamically stable and isotropic systems of oil, water, surfactant and cosurfactant mixture with a droplet size usually in the range of 10-100 $\mathrm{nm}$. These systems, by virtue of their low globule size and ability of improving aqueous solubility of poor water soluble drug like Curcumin, are widely explored as nasal drug delivery system to enhance CNS uptake (Patel, Mandal, Rajesh, 2012). Addition of mucoadhesive agent such as a polyelectrolyte polymer further helps to increase the retention time due to the electronic interaction between mucin of nasal mucosa and the polymer of formulation which in turn further facilitates the absorption (Elshafeey, Bendas, Mohamed, 2009; Harris et al., 1988). Evidences of intranasal drug delivery systems formulated using mucoadhesive agent and its benefits in enhancing noseto-brain drug transport have been reported in various literatures (Pathak et al., 2014; Mandal, Mandal, 2010). Also, several literatures revealed that targeting brain through nasal route is possible due to the unique noseto-brain connection (Tomoyuki et al., 2007). Also for the intranasal delivery, an optimal formulation with minimum number of experiments and low globule size to provide higher $\%$ release, an adequate permeation rate in a short time is required.

Thus, RSM with Box-Behnken design was used in this study to obtain the optimal CME and evaluate the influence of formulation composition and their proportion on drug permeation capacity through sheep nasal mucosa (Chopra et al., 2007; Gannu, Rao, 2012). In this study, the amounts of oil (X1), mixture of surfactant and cosurfactant (X2) and aqueous solution of sodium hyaluronate (X3) were selected as independent variables. The Box-Behnken design was used to investigate the main effects, interaction effects and quadratic effects of the individual independent factors on average globule size (Y1), polydispersity index (PdI) (Y2), flux (Y3), viscosity (Y4) and \% drug release after $10 \mathrm{~h}$ (Y5). CMME may result in effective noseto-brain transport and greater distribution of curcumin within and into the brain which may help to maximize the therapeutic index of the drug and may open newer approach of treating PD.

In our previous study the dissolution of Curcumin was enhanced through the developed microemulsion (Mandal, Mandal, Patel, 2013). Hence, the objective of this investigation is to explore the unique nose to brain connection through the optimal CMME and also to perform the comparative neuroprotective effect of curcumin in MPTP induced mice model of Parkinson's disease.

\section{MATERIAL AND METHODS}

\section{Material}

Curcumin was received as a gift sample from Arjuna Natural Extracts Pvt. Ltd., Hyderabad, India. Cremophor RH 40 and Cremophor EL were obtained from Colorcon Asia (Mumbai, India). Labrafac CM10, Transcutol HP, Transcutol P, Maisine 35-1, Labrafil M 1944 CS and Labrafac PG were received as gift sample from Gattefosse, France. MPTP-HCl was purchased from Sigma-Aldrich (St. Louis MO, USA).Capmul MCM, Capmul MCM EP, Accenon CC, Captex-355 and Labrafac were procured as gift sample from Abitec Corporation, Jackson St 
Janesville, USA. Isopropyl myristate, Tween 60, Isopropyl alcohol, Acetonitrile and Methanol wereboughtfrom Gujaratchemicalcorporations (Vadodara,India). Sodium hyaluronate, Oleic acid, Tween-60, Ethyl oleate and PEG 400 were bought from Chemdyes (Mumbai, India). All other chemicals were reagent grade.

\section{METHODS}

\section{Selection of formulation compositions}

For o/w microemulsion, selection of oil as internal phase was mainly based on the drug solubility capacity and biocompatibility as mentioned in the literature (Mandal, Mandal, 2010). Different oils like isopropyl myristate, Capmul MCM EP, Capmul MCM Labrafac PG, Oleic acid, Labrafil M 1944CS and Labrafil M 2125CS were screened for drug solubility study. Selection criteria for surfactant were its HLB value, drug solubility and nontoxic nature. Surfactants having HLB value ranging in between 12 to 18 like Tween-60, Tween-80, Captex-355, Accenon CC and Cremophor EL were screened for drug solubility study. Screening of co-surfactants was based on their ability to form stable and clear microemulsion at a minimum concentration and several co-surfactants like ethanol, Isobutyl alcohol, isopropyl alcohol and Transcutol HP were screened. The pseudo-ternary phase diagrams of oil, surfactant: co-surfactant $\left(\mathrm{S}_{\text {mix }}\right)$ and water were developed using distilled water titration method (Mandal, Mandal 2010). The mixture of oil and at different ratio of $\mathrm{S}_{\text {mix }}(1: 1,1.5: 1,2: 1,2.5: 1,3: 1$ and 3.5:1) at certain volume ratio were titrated with distilled water in drop wise manner. Using Chemix software, microemulsion regions were determined and $\mathrm{S}_{\text {mix }}$ showing maximum microemulsion region was used for the development of microemulsion (Mandal, Mandal, 2010).

\section{Preparation of formulations}

Box-Behnken design was used to develop CMMEs in which three different independent variables i.e., amount of Capmul MCM (X1), $\mathrm{S}_{\text {mix }}$ (Accenon CC: Transcutol HP, $\mathrm{X} 2$ ) and amount of sodium hyaluronate (X3, in terms of \% $\mathrm{w} / \mathrm{v}$ ) with their levels was set according to the preliminary experiments. A suitable polynomial model was selected based on the significant terms $(p<0.05)$, the least significant lack of fit, the multiple correlation coefficient $\left(\mathrm{R}^{2}\right)$ and adjusted multiple correlation coefficient (adjusted $\mathrm{R}^{2}$ ) provided by Design-Expert ${ }^{\circledR}$ software (Stat-Ease, Inc., Minneapolis, Minnesota, USA) (Gannu et al., 2010). The default model in the design of the experimental space was quadratic and the upper and lower levels are demonstrated in Table I.

On the basis of the Box-Behnken design model provided by Design-Expert ${ }^{\circledR}$ Software (Stat-Ease, Inc., Minneapolis, Minnesota, USA), a total 15 model CMMEs were randomly arranged. The compositions of all model CMMEs are summarized in Table I. $\mathrm{S}_{\text {mix }}$ (2.5:1 of Accenon CC and Transcutol HP) was mixed well with drug dissolved Capmul MCM part. The above mixture was then titrated with different aqueous sodium hyaluronate concentration with mild and continuous stirring by magnetic stirrer at room temperature (Mandal, Mandal, Patel, 2013) and finally average globule size, polydispersity index (PdI), Flux, viscosity and \% drug release after $10 \mathrm{~h}$ were determined. Curcumin solution (CPG, $3 \mathrm{mg} / \mathrm{mL}$ ) was prepared by addition of Curcumin $(30 \mathrm{mg})$ to $8 \mathrm{~mL}$ of distilled water and ethanol $(6: 4)$ with continuous stirring. The $\mathrm{pH}$ was adjusted to $6.8 \pm 0.25$ using glacial acetic acid (approximately $0.06 \mathrm{~mL} / \mathrm{mL}$ ). The mixture was stirred for $10 \mathrm{~min}$ and a clear solution was obtained. Final volume was made up to $10 \mathrm{~mL}$ using distilled water. $\mathrm{pH}$ of the final formulations was also checked.

\section{Optimization}

Responses like average globule size, polydispersity index (PdI), Flux, viscosity and \% drug release after $10 \mathrm{~h}$ were selected for both numerical and graphical optimization. It was decided to choose maximization for flux, \% release and minimization for average globule size and PdI with suitable viscosity in order to obtain an optimized formulation. On a contour plot, visually search for the best compromise which stands for the formulation with desirable values for all responses were visually searched. Finally, for verification, three optimized CMME batches were prepared experimentally and the responses i.e., average globule size, polydispersity index (PdI), flux, viscosity and $\%$ drug release for all the batches were determined. Flux i.e., amount of drug release per area of permeation was quantified from the ex vivo permeation study through sheep nasal mucosa.

\section{Evaluation of dependent variables}

Globule size and Zeta potential of CMMEs were determined by Zetasizer (Nano ZS; Malvern Instruments Inc, Malvern, UK). The viscosity of CMMEs determined using Brookfield viscometer (HVDVII, USA). The sample was sheared using spindle no. 43 at $30 \mathrm{rpm}$ and at room temperature. Data was taken after $30 \mathrm{~s}$ of viscosity 
TABLE I - Details of the levels, compositions and responses of model formulations (CMME) provided by Box-Behnken design

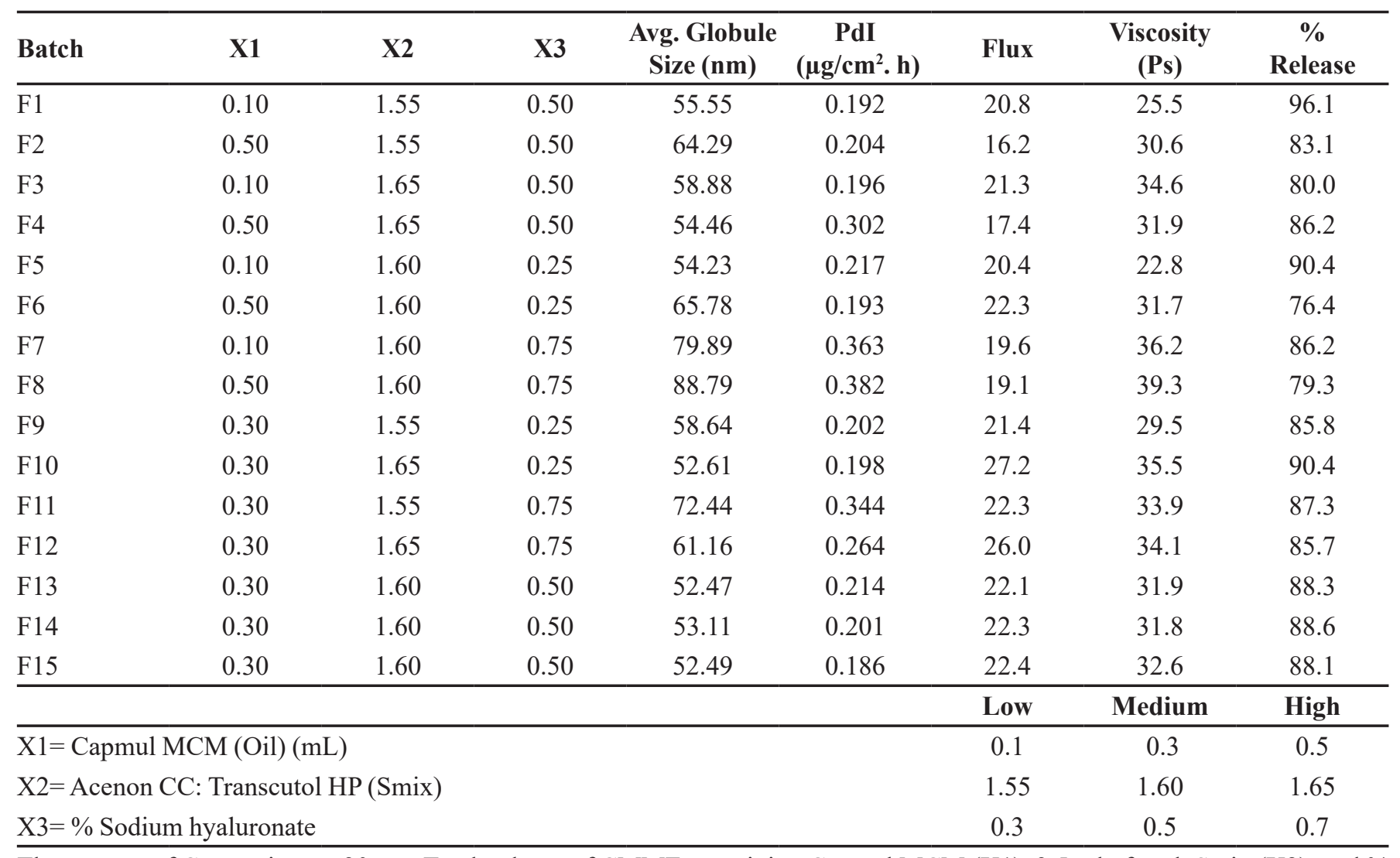

The amount of Curcumin was $30 \mathrm{mg}$. Total volume of CMME containing Capmul MCM (X1), $3.5 \mathrm{ml}$ of each Smix (X2), and \% Sodium hyaluronate(X3) was $10 \mathrm{~mL}$.

measurement. All experiments were repeated three times (Mandal, Mandal, 2010).

In vitro comparative release of Curcumin from CMME and CPG was performed using USP-II dissolution apparatus. $10 \mathrm{ml}$ of CMME $(\approx 30 \mathrm{mg}$ of curcumin $)$ was taken in dialysis membrane $(10,000 \mathrm{D})$ and was kept in $900 \mathrm{ml}$ of the dissolution medium i.e., phosphate buffer (pH-6.4) at $37 \pm 0.5{ }^{\circ} \mathrm{C}$ and $50 \mathrm{rpm}$ (Mandal, Mandal, Patel, 2013). At predetermined time interval, aliquots of $5 \mathrm{ml}$ in each test time point was withdrawn from receptor compartment and replaced with same amount of freshly prepared dissolution medium equilibrated at $37 \pm 0.5^{\circ} \mathrm{C}$. The samples were the analysed for drug release by UV-VIS Spectrophotometer (Mandal, Mandal, Patel, 2013).

Comparative permeability of CMME and CPG through excised sheep nasal mucosa was determined using Franz diffusion cell having an effective diffusion area of $12.56 \mathrm{~cm}^{2}$ and volume $25 \mathrm{~mL}$ (Patel, Mandal, Rajesh, 2012). The prepared nasal mucosa of $12.56 \mathrm{~cm}^{2}$ was mounted on to the receptor compartment having 25 $\mathrm{mL}$ of diffusion medium (Saline phosphate buffer, $\mathrm{pH}-6.4$ ) for $15 \mathrm{~min}$. The donor compartment was fixed to it and was loaded with formulations ( $\approx 6 \mathrm{mg}$ of curcumin). Diffusion was done at $37 \pm 0.5{ }^{\circ} \mathrm{C}$ and $50 \mathrm{rpm}$. At predetermined intervals, up to $10 \mathrm{~h}$, aliquot of $0.5 \mathrm{~mL}$ was withdrawn from the receptor medium and was analyzed by UV-VIS Spectrophotometer (Mandal, Mandal, 2010; Mandal, Mandal, Patel, 2013). Each data point represented the average of three determinations. Flux i.e., \% drug release per unit area of the sheep nasal mucosa per unit time was also determined.

\section{Data analysis}

The formulation variables i.e., Independent variables (X1, X2 and X3) and responses or dependent variables (average globule size, PdI, viscosity, flux and \% drug release) of all CMMEs were treated by the DesignExpert ${ }^{\circledR}$ software. The linear, quadratic, and cubic models was used to describe the relation between independent and dependent variables for the constrained mixture designs consisting of three components. The significant terms were set at a $p$-value of less than 0.05 . The suitable fitting model was selected based on the comparisons of 
several statistical parameters including the $p$ - value of the model ( $p$-value must be less than 0.05), $p$-value of lack of fit ( $p$-value must be greater than 0.05$)$, the multiple correlation coefficient $\left(R^{2}\right)$, adjusted multiple correlation coefficient (adjusted $R^{2}$ ), and the coefficient of variation proved by the Design-Expert ${ }^{\circledR}$ software (Chopra et al., 2007; Gannu, Rao, 2012).

\section{Nasal ciliotoxicity}

Nasal ciliotoxicity studies were carried out using excised sheep nasal mucosa. In brief, excised sheep nasal mucosa except septum was collected from slaughter house in saline phosphate buffer ( $\mathrm{pH}$ 6.4). Then three different nasomucosal parts (S1, S2 and S3) having same thickness were mounted on Franz diffusion cell and then were exposed with $2 \mathrm{ml}$ of CMME (Test sample, $2.86 \mathrm{mg} / \mathrm{mL}$ curcumin), saline (Negative control) and isopropyl alcohol, a serious nasal mucociliary toxicity agent (Positive control) for $2 \mathrm{~h}$ respectively. All three nasal samples were rinsed with distilled water. The mucocilia was then examined with an optical microscope (Nikon Fx-35A, Japan) after the sample stained with hematoxylin and eosin (Mandal, Mandal, 2010).

\section{In-vitro mucoadhesion study}

The mucoadhesive potential of developed nasal formulations in terms of residence time was evaluated by the reported method (Pathak et al., 2014). Briefly, $100 \mathrm{mg}$ of CPG and CMME was placed at the center of separate agar plates (1\% w/w, prepared in PBS, $\mathrm{pH} 6.4)$. After 5 $\mathrm{min}$, the agar plates were attached to USP disintegration test apparatus and moved up and down in PBS at $37^{\circ} \mathrm{C}$ \pm 1 . The time taken by the formulations to separate from the agar plates was noted visually as residence time of the formulations.

\section{In vivo experimental study}

All animal experiments were approved by the Institutional Animal Ethical Committee (CPCSEA No. 984/14/10/CPCSEA), Government of India, New Delhi, India. Male C57BL/6 mice (20-25 g; 8-12 weeks old) were used in the present study. The mice were maintained at temperature $\left(25 \pm 2{ }^{\circ} \mathrm{C}\right)$ and humidity $(45 \pm 5 \%)$ and were supplied with standard laboratory diet and reverse osmosis (RO) water ad libitum on a $12 \mathrm{~h}$ light/dark cycle. Mice were assigned to five groups with six animals in each group.

Group I - Saline treated (Normal control).
Group II - MPTP control $(4 \times 20 \mathrm{mg} / \mathrm{kg} /$ day $) .0 .2 \%$ of MPTP-hydrochloride was prepared using $0.9 \% \mathrm{w} / \mathrm{v} \mathrm{NaCl}$ solution. Four doses of $20 \mathrm{mg} / \mathrm{kg}$ MPTP solution (total dose of $80 \mathrm{mg} / \mathrm{kg}$ of body weight) at two hour intervals were administered through intraperitoneal route.

Group III- Intranasal administration of optimized CMME at $2.86 \mathrm{mg}$ of curcumin $/ \mathrm{kg}$ of body weight for 14 consecutive days to already MPTP $(4 \times 20 \mathrm{mg} / \mathrm{kg} /$ day $)$ treated animal in the same above method (as described for Group II).

Group IV- Animals were first treated with MPTP $(4 \times 20$ $\mathrm{mg} / \mathrm{kg} /$ day) in the same above method followed by intranasal applied plain drug solution (CPG) at $2.86 \mathrm{mg}$ of curcumin $/ \mathrm{kg}$ of body weight for 14 consecutive days.

Group V-Animals were treated with only $2.86 \mathrm{mg}$ of curcumin $/ \mathrm{kg}$ of body weight for 14 consecutive days.

35-40 $\mu \mathrm{L}$ of the developed nasal formulations containing curcumin equivalent to $2.86 \mathrm{mg} / \mathrm{kg}$ were administered intranasally with the help of a micropipette $(200 \mu \mathrm{L})$ attached to low density polyethylene tubing with internal diameter $0.1 \mathrm{~mm}$ at the delivery site (Elshafeey et al., 2009). Ventral mid brain and striatum of the three experimental animals was dissected on $15^{\text {th }}$ day and $21^{\text {st }}$ day of study after euthanized with ketamine and then the brain tissues were preserved at $-80 \pm 1{ }^{\circ} \mathrm{C}$ for further investigation.

\section{Determination of muscular coordination by Rota- rod test}

The motor coordination activity was assessed by rota-rod apparatus (Baroda Pvt. Ltd. India) as described in the literature (Hiromi et al., 2010). The instrument was divided into four compartments and has a rotating rod of $30 \mathrm{~mm}$ diameter. The rotation speed of the rod was set at $20 \mathrm{rpm}$ and four mice at a time were tested for the total duration of $180 \mathrm{~s}$. The apparatus was designed to record the time automatically when a mouse falls off from the rotating shaft. The time in seconds spent on the rod was recorded (retention time) for each mice and the average was used as a measure of motor function at $15^{\text {th }}$ and $21^{\text {st }}$ day.

\section{Assessment of gross behavioural activity by open field test}

The open field test was performed as described in the literature (Basso, Beathie, Bresnahan, 1995; Holcomb et al., 2006). The open-field apparatus consists of a box $(26 \times 26 \times 39 \mathrm{~cm})$ with clear plexi-glass walls and floor. Briefly, the animals were placed in the centre of a box 
with the floor lined by equal segments, and the motor (the number of crossed segments) activity was monitored for 5 min. An automated sensor system, consisting of 16 photo beams per side measured the fine movement, expressed as total ambulation time at $15^{\text {th }}$ and $21^{\text {st }}$ day.

\section{Quantification of dopamine and its metabolites in brain by HPLC}

The assay of dopamine (DA) and its metabolites like dihydroxyphenyl acetic acid (DOPAC) and homovanillic acid (HVA) was quantified by using HPLC-fluorescence detection as described in the literature (Liu, Ma, Shi, 2006; Barbiero et al., 2011). Briefly, striatum after was homogenized in phosphate buffer ( $\mathrm{pH}-6.4)$ in tissue homogeniser and the homogenate was centrifuged at $15,000 \mathrm{rpm}\left(-4{ }^{\circ} \mathrm{C}\right)$ for $300 \mathrm{~min} .20 \mu \mathrm{L}$ samples i.e., filtered supernatant through $0.45 \mu \mathrm{m}$ membrane filter was injected on the column. The mobile phase was consisted of acetonitrile and $0.1 \%$ orthophosphoric acid $(50: 50 \mathrm{v} / \mathrm{v})$. The mobile phase flow rate was set at $1 \mathrm{~mL} /$ min. The DA, DOPAC and HVA signals were detected at $282 \mathrm{~nm}$ and $324 \mathrm{~nm}$ as the excitation wavelength and an emission wavelength respectively. The quantification of DA, DOPAC and HVA was carried out by measuring the chromatographic peak areas and results were expressed as micrograms per gram wet tissue at $7^{\text {th }}$ day as well as $15^{\text {th }}$ day.

\section{Statistical analysis}

All data are reported as mean $\pm \mathrm{SD}$ and the difference between the groups were tested using Student's t-test at the level of $\mathrm{P}<0.05$. More than two groups were compared using ANOVA and differences greater at $\mathrm{P}<0.05$ were considered significant.

\section{RESULTS AND DISCUSSION}

\section{Selection of formulation composition and formulation development}

Capmul MCM was selected as internal phase due to its non-toxicity to nasal mucosa and relatively more Curcumin solubilising capacity. Accenon CC basing on its HLB value with minimum drug solubility was selected as surfactant. This was done with an intention to obtain more trafficking of drug to obtain more sustained release. Transcutol HP was selected as cosurfactant since with minimum concentration it developed stable microemulsion and also due to the biocompatibility to nasal mucosa and compatibility to other formulation compositions including Curcumin as determined through FTIR.

Results of Pseudo-ternary phase diagrams showed that formulation with $\mathrm{S}_{\text {mix }}(2.5: 1)$ was having more microemulsion existing zone. Hence, from the drug solubilising and pseudo-ternary phase diagrams data, Capmul MCM, Transcutol HP and Accenon CC were selected as oil phase, co-surfactant and surfactant respectively to develop microemulsion.

In order to quantify the effect of independent variables on the permeation parameters and the properties of CMMEs, the values of the dependent variables (average globule size, PdI, viscosity, flux and \% release) and three independent variables Capmul MCM concentration (X1), concentration of $\mathrm{S}_{\text {mix }}(\mathrm{X} 2)$ and concentration of aqueous sodium hyaluronate solution (X3) were statistically analyzed based on the RSM, using a computer program provided by the Design- Expert ${ }^{\circledR}$ software. The results of multiple regression analysis are summarized in Table II.

The probability ( $p$-value) of the models was less than 0.05 and the $p$-value of the lack of fit was greater than 0.05 , indicating that the selected model could well

TABLE II - Statistical parameter of responses determined by multiple regression Regression Analysis

\begin{tabular}{lccccc}
\hline \multirow{2}{*}{ Regression Coefficient } & \multicolumn{5}{c}{ Coefficient Estimate } \\
\cline { 2 - 6 } & Globule size & PdI & Viscosity & Flux & \% Release \\
\hline Capmul MCM (X1) & 5.14 & 2.38 & 0.87 & 0.87 & -4.56 \\
Smix (X2) & -8.32 & -1.23 & -1.66 & 1.25 & +1.87 \\
\% Sod. hyaluronate (X3) & 7.77 & 4.23 & 2.33 & -1.12 & -2.77 \\
Model (p Value) & 0.0003 & 0.0014 & $<0.0001$ & 0.0212 & $<0.0001$ \\
Coefficient of variation & $2.28 \%$ & $3.09 \%$ & $1.33 \%$ & $8.40 \%$ & $0.86 \%$ \\
$\mathrm{R}^{2}$ & 0.987 & 0.977 & 0.999 & 0.928 & 0.999 \\
Adjusted ${ }^{2}$ & 0.964 & 0.935 & 0.998 & 0.799 & 0.997 \\
Lack of Fit $(p$ Value) & 0.0540 & 0.0917 & 0.289 & 0.877 & 0.0580 \\
\hline
\end{tabular}


describe the relationship between the independent and dependent variables. The values of the coefficients X1, $\mathrm{X} 2$ and $\mathrm{X} 3$ were represented to the effect extent of these independent variables on the response. The relationship between the independent and dependent variables was elucidated using response surface plots. It was also found that average globule size, PdI, viscosity, flux and \% release of Curcumin was remarkably influenced by the main effect and interaction effect of various independent variables as shown in Table II.

Analysis for all responses showed that quadratic model was the most suitable one $(p<0.05)$. By running ANOVA, the final equations for all responses i.e., Globule size, PdI, Viscosity, Flux and \% Release in their respective coded values (A, B and C) were obtained as follows. The statistical analysis showed that A, B, C and their interactions are significant model terms for response 1 (Globule size) and response 2 (PdI), $\mathrm{C}$ is significant model terms for response 3 (viscosity) and $\mathrm{B}$ are significant model terms for response 4 (Flux) and A, C, AC are significant model terms for response 5 ( $\%$ Release).

Globule Size $=+154.84+5.14 \times \mathrm{A}-8.32 \times \mathrm{B}+$

$7.77 \times \mathrm{C}+1.86 \times(\mathrm{A} \times \mathrm{B})+1.79 \times(\mathrm{B} \times \mathrm{C})+$

$9.84 \times(\mathrm{A} \times \mathrm{C})+3.15 \times \mathrm{A}^{2}-0.98 \mathrm{~B}^{2}+5.47 \times \mathrm{C}^{2}$

The globule size of microemulsion formulation is a crucial parameter as because it influences drug release rate and hence the in vivo profile of the drug. From equation 1 , globule size was influenced by oil, Surfactant-cosurfactant concentration ratio $\left(\mathrm{S}_{\text {mix }}\right)$ and mucoadhesive polymer. This may be due to the fact that $\mathrm{S}_{\text {mix }}$ at its increased concentration could able to reduce the interfacial tension between oil and aqueous phase. But at higher amount of oil with same $\mathrm{S}_{\text {mix }}$ concentration, the lipophilicity of oil was not completely being masked by $\mathrm{S}_{\text {mix }}$ resulting into more interfacial tension and increased globule size. Mucoadhesive polymer also found to increase the globule size which may be due to the fact that it was capable of absorbing water and swell which in turn disturb the hydrophilic-lipophilic balance of the system.

PdI Value $=+74.79+2.38 \times \mathrm{A}-1.23 \times \mathrm{B}+4.23 \times \mathrm{C}+$ $0.86 \times(\mathrm{A} \times \mathrm{B})+1.33 \times(\mathrm{B} \times \mathrm{C})+6.79 \times(\mathrm{A} \times \mathrm{C})+$ $0.97 \times \mathrm{A}^{2}-0.98 \mathrm{~B}^{2}+0.86 \times \mathrm{C}^{2}$

Polydispersity index (PdI) indicates the particle size distribution of the formulation. $\mathrm{S}_{\text {mix }}$ was capable reducing the interfacial tension between Capmul MCM and external aqueous phase as shown in equation 2 . The effect of stabilizers concentration on particle size and PdI value of CMME is depicted in equation 2. Both the particle size and PdI value decreased first and then increased with the increase of $\mathrm{S}_{\text {mix }}$ concentration which may be due to destabilisation of the system due to the stabilization effect. However, the surface area of microemulsion particles is limited, resulting in a limited amount of stabilizer adsorbed. In addition, excess surfactants tend to form micelles and increase solubility of water insoluble Curcumin, which may lead to Ostwald ripening, resulting in an increase in particle size and PdI.

Viscosity $=+35.14+0.87 \times \mathrm{A}-1.66 \times \mathrm{B}+2.33 \times \mathrm{C}-$ $1.12 \times(\mathrm{A} \times \mathrm{C})-0.59 \times \mathrm{A}^{2}+1.44 \times \mathrm{C}^{2}+1.89 \times$ $(\mathrm{A} \times \mathrm{C})$

Microemulsion viscosity increased with an increase in oil and mucoadhesive polymer concentration as shown in equation 3 . This may be due to the fact that mucoadhesive polymer absorbs aqueous part of the formulation and swelled. Free water concentration reduced and hence viscosity of the microemulsion automatically increased. Interaction effect of Capmul MCM and \% sodium hyaluronate got positive effect while interaction of oil and mixture of surfactant and cosurfactant showed opposite effect to that of mucoadhesive polymer alone.

Flux $=+45.61+0.87 \times \mathrm{A}+1.25 \times \mathrm{B}-1.12 \times(\mathrm{C})+$
$0.78(\mathrm{~A} \times \mathrm{B})-1.47(\mathrm{~A} \times \mathrm{C})$

Oil (A) and $\mathrm{S}_{\text {mix }}$ (B) concentration showed relatively significant effect on flux as shown in equation 4 . Accenon CC and Transcutol HP reduce the globule size and hence surface area increased which in turn increases the permeation rate through nasal mucosa or flux by altering the fluidization of lipid of the nasal mucosal layer. As mentioned above, the average globule size of CMME influences drug release rate and absorption.

$$
\begin{aligned}
& \% \text { Release }=+134.4-4.56 \times \mathrm{A}+1.87 \times \mathrm{B}-2.77 \times \mathrm{C} \\
& +0.55 \times(\mathrm{A} \times \mathrm{B})-1.83 \times(\mathrm{A} \times \mathrm{C})+0.71 \times(\mathrm{B} \times \mathrm{C})+ \\
& 1.41 \times \mathrm{A}^{2}-0.89 \times \mathrm{C}^{2}
\end{aligned}
$$

Drug release from the formulation was heavily but negatively influenced by Capmul MCMand sodium hyaluronate, while Accenon CC and Transcutol HP showed positive effect as shown in the equation 5. Capmul MCM and sodium hyaluronate concentration in the formulation reduced the drug release from the formulation which may be due to their reservoir property of oil and enhanced viscosity due to polymer. Surfactant and cosurfactant concentration increases the drug release due to their water 
solubility enhancing property of poorly water soluble drugs like curcumin. The aim of optimization was to obtain the defined targets for all responses simultaneously with respect to the predefined constraints. Overlay plots of all responses for predicted formulations are depicted in Figure 1.

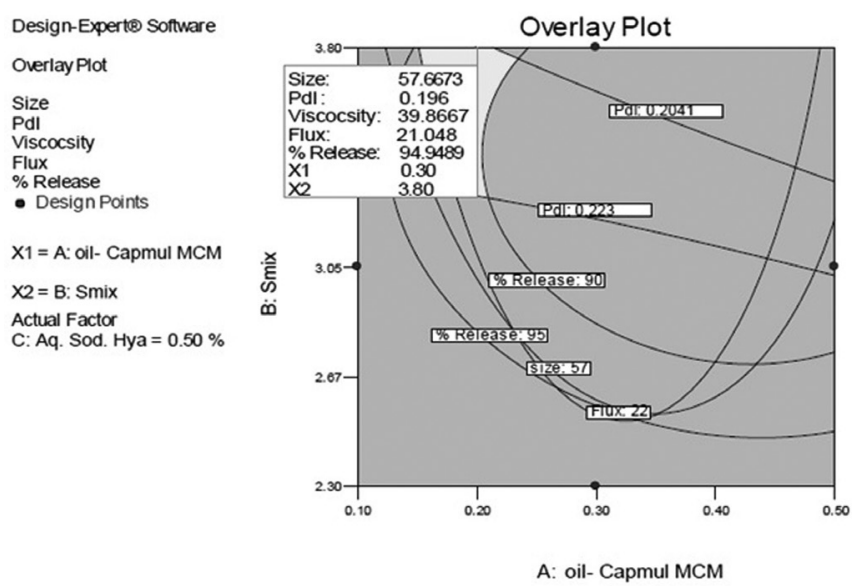

FIGURE 1 - Overlay plots of response (Globule size, PdI, Viscosity, Flux and \% Release) of predicted formulation.

The grey region stands for formulations with minimum globule size, maximum release and maximum flux. In order to confirm the desirability of provided optimized formulations, five formulations were prepared experimentally and all five responses i.e., average globule size, PdI, viscosity, flux and \% release were evaluated as given in Table III. It was observed that the experimentally obtained data and the predicted responses were significantly same and therefore, the optimization process was verified.

CMMEs developed with $0.5 \% \mathrm{w} / \mathrm{v}$ of sodium hyaluronate, $0.3 \mathrm{~mL}$ Capmul CMC, $3.70 \mathrm{~mL}$ of $\mathrm{S}_{\text {mix }}$ (Accenon CC and Transcutol HP at 2.5:1 ratio) showed the lowest globule size and PdI, highest flux, optimum viscosity and highest drug release as shown in Table IV and hence was considered as optimized formulation. This was also predicted by the application of experiment design methods since the developed CMME showed minimum difference between the predicted and observed responses.

\section{Evaluation of microemulsion formulation}

Optimal CMME was found to be transparent, stable with viscosity ranging from $40.22 \times 10^{3} \mathrm{cPs}$ to $46.18 \times 10^{3}$ cPs at $25^{\circ} \mathrm{C}$ with globule size $57.6 \pm 3.46 \mathrm{~nm}$ as shown in Figure 2. Data of Zeta potential was $-16.28 \pm 2.94 \mathrm{mV}$ as shown in Figure 3 indicates stability of formulation since no aggregation due to repulsion and attraction will be observed.

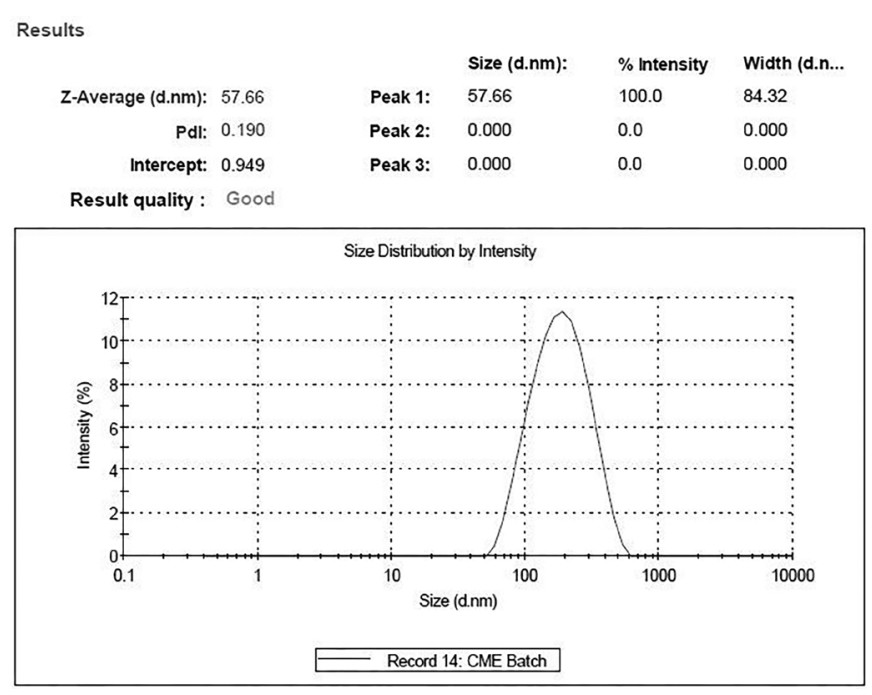

FIGURE 2 - Result of average globule size and PdI indicating the nanosize with narrow size distribution of developed CMME.

Results of globule size and PdI value $(0.190 \pm 0.19)$ indicates that the formulation was monodisperse and of nanosize which may help in the intranasal permeation

TABLE III - Desirability confirmation of Box-Behnken design through the comparision of predicted and observed results of size, PdI, viscosity, flux and \% release of CMME

\begin{tabular}{lccccccccccccc}
\hline \multirow{2}{*}{ Sr No. } & \multicolumn{3}{c}{ Component } & \multicolumn{3}{c}{$\begin{array}{c}\text { Globule size } \\
(\mathbf{n m})\end{array}$} & \multicolumn{2}{c}{ PdI } & \multicolumn{2}{c}{$\begin{array}{c}\text { Flux } \\
{\left[\boldsymbol{\mu g} /\left(\mathbf{c m}^{2} \mathbf{h}\right)\right]}\end{array}$} & \multicolumn{2}{c}{ Viscosity (Ps) } & \multicolumn{2}{c}{$\begin{array}{c}\text { \% Release } \\
(\text { After 10h) }\end{array}$} \\
\cline { 2 - 14 } & $\mathbf{O}$ & $\mathbf{S}_{\text {mix }}$ & $\mathbf{P}$ & Pre. & Obs. & Pre. & Obs. & Pre. & Obs. & Pre. & Obs. & Pre. & Obs. \\
\hline 1 & 0.30 & 3.80 & 0.50 & 58.6 & 57.4 & 0.198 & 0.208 & 21.0 & 19.4 & 39.8 & 38.2 & 95.9 & 92.6 \\
2 & 0.31 & 3.80 & 0.52 & 58.4 & 57.5 & 0.205 & 0.216 & 20.9 & 20.0 & 39.5 & 37.1 & 91.3 & 93.0 \\
3 & 0.31 & 3.75 & 0.50 & 58.9 & 58.0 & 0.198 & 0.202 & 20.8 & 20.2 & 39.5 & 38.0 & 94.4 & 91.6 \\
4 & 0.30 & $\mathbf{3 . 7 0}$ & 0.50 & $\mathbf{5 6 . 7}$ & $\mathbf{5 6 . 3}$ & $\mathbf{0 . 1 9 8}$ & $\mathbf{0 . 1 9 5}$ & $\mathbf{2 0 . 6}$ & $\mathbf{2 1 . 8}$ & $\mathbf{3 9 . 3}$ & $\mathbf{3 8 . 7}$ & $\mathbf{9 2 . 1}$ & $\mathbf{9 1 . 8}$ \\
5 & 0.30 & 3.80 & 0.50 & 56.6 & 58.1 & 0.212 & 0.227 & 22.0 & 2.89 & 40.2 & 42.1 & 87.5 & 88.9 \\
\hline
\end{tabular}

(Pre.- Predicted and Obs.- Observed) 


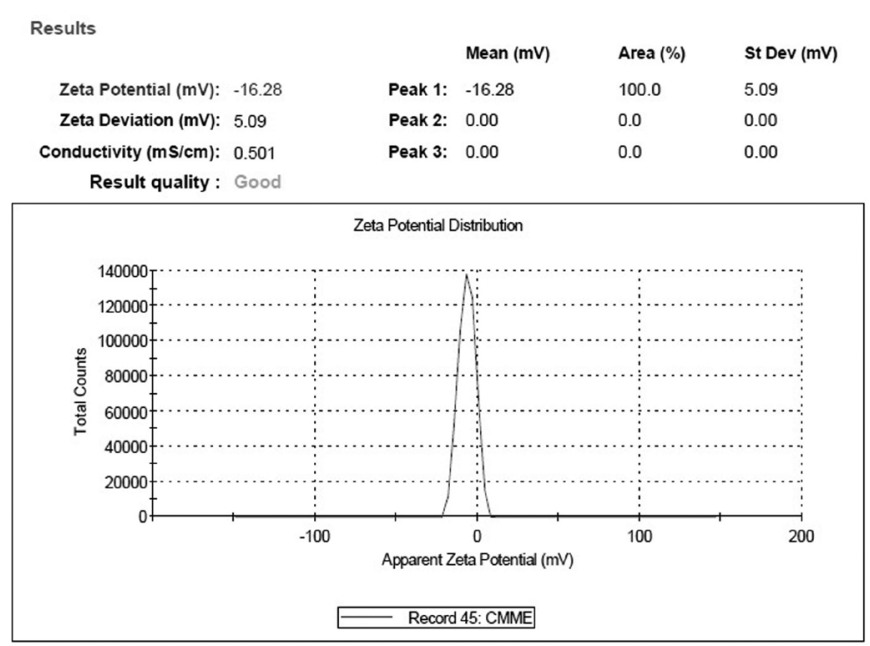

FIGURE 3 - Zeta potential of CMME showing its stability during storage.

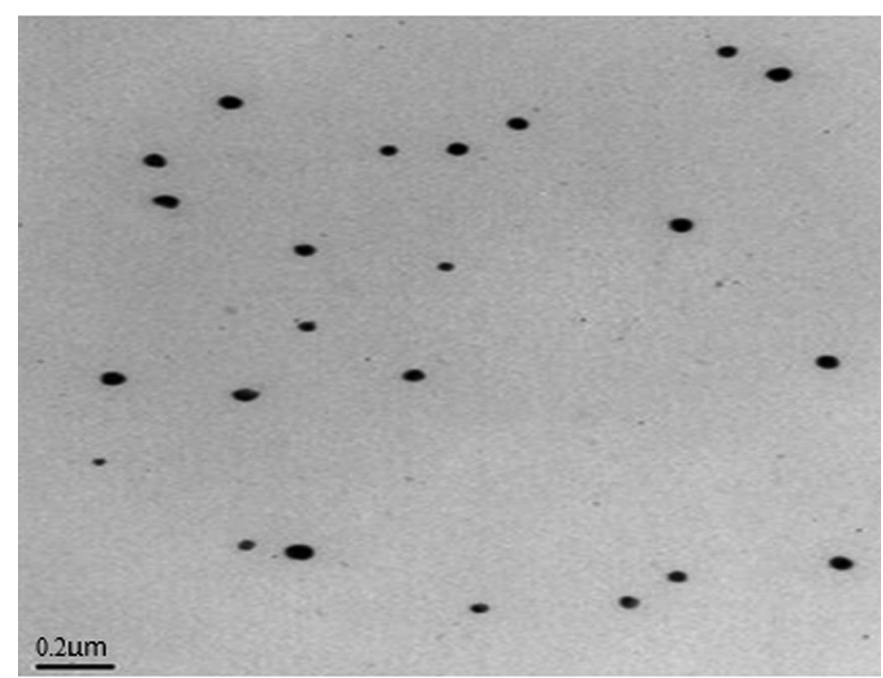

FIGURE 4 - TEM result of the optimized CMME indicating the narrow particle size with uniform distribution.

which is supported by the TEM results as shown in Figure 4.

Viscosity data also confirmed the residence of the microemulsion on the nasal mucosa. However, the obtained result indicated that the physical properties of CMMEs were significantly influenced by the combinations of independent variables.

\section{In vitro release and ex vivo permeation study}

Release study showed that $93.81 \pm 2.68 \%$ of drug was sustained released from CMME for $10 \mathrm{~h}$. From the data, it was observed that the release rate was decreased with increase amount of independent variables like oil and mucoadhesive polymer. This may be due to the fact that partitioning of drug was more profound when the amount of the internal phase and mucoadhesive polymer were increased which in turn due to the solubility of drug in these vehicles. This can be explained by Noyes-Whitney equation, where the rate of dissolution increases with a decrease in the particle size, because of the resultant enhancement in the surface area and decrease in the diffusion layer thickness. The flux of $22.17\left[\mu \mathrm{g} /\left(\mathrm{cm}^{2} \mathrm{~h}\right)\right]$ revealed the quick permeation through nasal mucosa which may be due to the relatively smaller globule size and cumulative penetration action of oil, surfactant and co-surfactant. The effect of oil, mixture of surfactant and co-surfactant and sodium hyaluronate on physical properties and drug penetration capacity of microemulsion was evaluated by RSM with Box-Behnken design and the results indicated that $\mathrm{CMME}$ system had a significant permeation enhancement effect for intranasal delivery of Curcumin. Moreover, the developed system can reduce the interface tension between nasal mucosa and vehicle and thus, they can achieve a faster permeation which will be supported by the viscosity of the formulation.

\section{Nasal ciliotoxicity}

Results of nasal ciliotoxicity studies as shown in the Figure 5 reveal the non-toxicityeffect of the developed CMME and hence it was found suitable for application on the nasal mucosa.

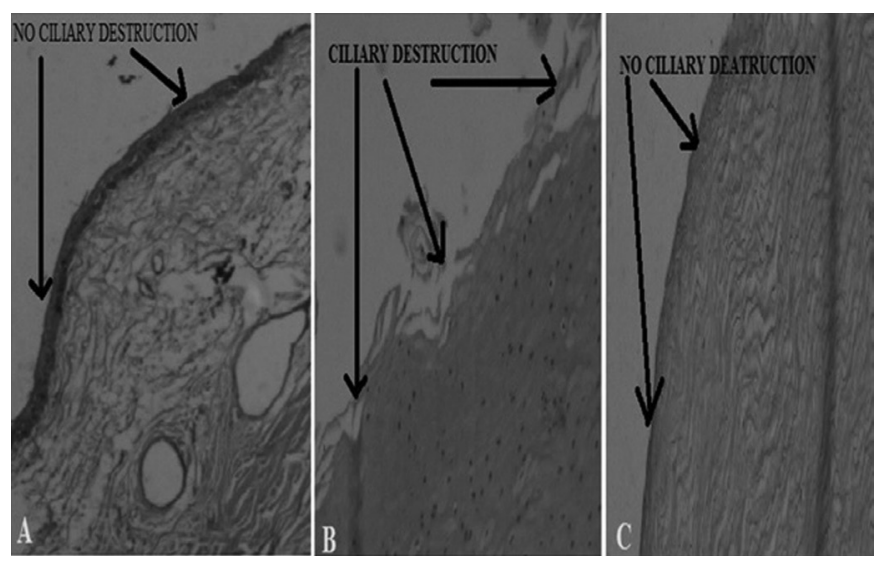

FIGURE 5 - Result of nasociliotoxicity study showing the non-toxicity of developed CMME.5[A], 5[B] and 5[C] are representative of saline, isopropyl alcohol and developed CMME treated mucosal part respectively.

\section{In-vitro mucoadhesion study}

The observed retention time for CMME and CPG were $17.0 \pm 1.5$ and $1.5 \pm 0.55 \mathrm{~min}$, respectively $(n=3)$. The retention time on agar plate showed by CMME was 
significantly higher than CPG. Sodium hyaluronate after hydration in the nasal ciliary microenvironment forms hydrogen bonds with the biological substrates through its carboxylic group (Morimoto et al., 1991). Thus, it can be concluded that the developed CMME will be able to increase the residence time of the formulation on the nasal mucosa which can be attributed due to presence of sodium hyaluronate $(0.5 \% \mathrm{w} / \mathrm{w})$ (Horvat et al., 2009; Pathak et al., 2014).

\section{In vivo study}

\section{Determination of muscular coordination and gross behavioural activity}

Figure 6 depicted significant $(p<0.05)$ reduction in motor performance in MPTP group as compared to normal control group as well as CPG. Treatment with Curcumin $(2.86 \mathrm{mg} / \mathrm{kg}$ ) through CMME in third group of animals significantly $(\mathrm{p}<0.05)$ improved the muscular coordination activity as compared to MPTP-alone group.Further, MPTP intoxication resulted in significant $(\mathrm{p}<0.05)$ decrease in total gross activity as compared to normal control group. Intranasal administration of CMME (2.86 mg curcumin per $\mathrm{kg}$ of body weight) significantly $(\mathrm{p}<0.05)$ improved the total gross activity when compared to MPTP-alone group as shown in Figure 6. although CPG initially showed improved muscular coordination and gross behavioural activity but subsequently not sustained the proposed beneficial effect since only mucoadhesive property of CPG on nasal mucosal membrane not able to alter the aqueous solubility of curcumin.

Protective effect of curcumin on gross neurological

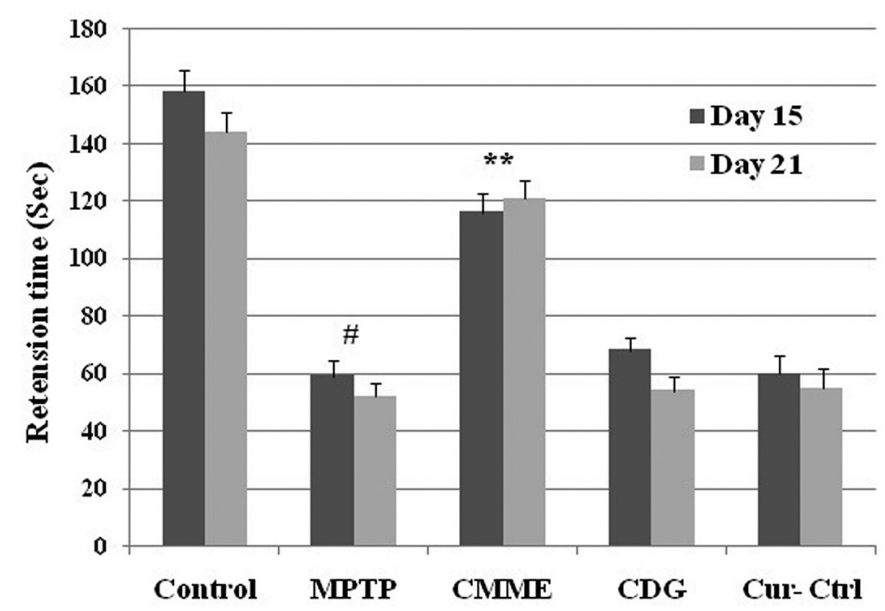

FIGURE 6 - Protective effect of CMME on motor coordination in rota-rod test. Data are expressed as mean $\pm \mathrm{SD}$ (06 per group). $\# \mathrm{P}<0.05$ normal control v/s MPTP and $* * \mathrm{P}<0.05 \mathrm{MPTP} \mathrm{v} / \mathrm{s}$ treated group. activity as assessed through an open field test. Steep reduction in total ambulation time was observed in MPTP-intoxicated group as compared to normal controls. Curcumin treatment significantly improved the total ambulation time as shown in Figure 7.

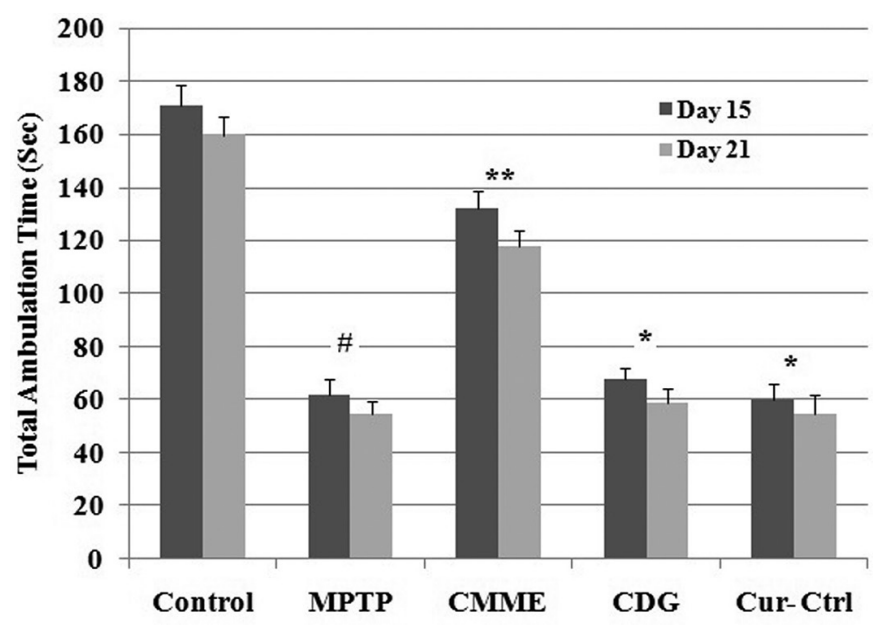

FIGURE 7 - Protective effect of Ibuprofen on gross neurological activity as assessed through an open field test. Values are expressed as mean $\pm \mathrm{SD}$ ( $\mathrm{n}=06$ per group.) Normal control v/s MPTP $(\# \mathrm{P}<0.05)$ and MPTP $\mathrm{v} / \mathrm{s}$ treated group $(* * \mathrm{P}<0.05)$.

\section{Quantification of DA and its metabolites}

MPTP intoxication significantly $(\mathrm{p}<0.05)$ decreased striatal DA content to $21.29 \%(6.53 \pm 0.86 \mu \mathrm{g} / \mathrm{g}$ of brain tissue) in comparison to normal control $(21.29 \pm 1.54 \mu \mathrm{g} / \mathrm{g}$ of brain tissue $\cong 100 \%$ ), which was then elevated to $55.37 \%$ $(13.67 \pm 0.58 \mu \mathrm{g} / \mathrm{g}$ of brain tissue) by curcumin treatment. In-addition, a concomitant elevation of DA turnover was also observed in MPTP induced mice which was significantly prevented on curcumin treatment as shown in Table IV.

Neuroprotective effect of curcumin against MPTP mediated dopaminergic neuroinflammation. Intranasal administration of curcumin significantly protected dopamine depletion with concomitant elevation in DA turnover compared to MPTP-treated mice. Values are expressed as mean $\pm \mathrm{SD}$ and statistically significant (\# $\mathrm{P}<0.05$ ) for normal control V/s MPTP and (* $\mathrm{P}<0.05)$ MPTP V/s curcumin treated groups $(n=6)$.

These findings suggestive of neuroprotective efficacy of curcumin via developed CMME through intranasal route. Furthermore, curcumin concentration was high in brain tissue for CMME than that of CPG ( $2007 \mathrm{ng} / \mathrm{g} \pm 37.88$ of brain tissue $\mathrm{V} / \mathrm{s} 301.4 \mathrm{ng} / \mathrm{g} \pm 21.21$ ) indicating the suitability of brain delivery of curcumin by CMME through nasal route over CPG. The mechanisms 
TABLE IV - Comparative neuroprotective effect of CMME in animal models

\begin{tabular}{lcccc}
\hline \multirow{2}{*}{ Groups } & DA & DOPAC & HVA & $\begin{array}{c}\text { Turnover } \\
\text { (DOPAC+HVA/DA) }\end{array}$ \\
\cline { 2 - 3 } & & $\boldsymbol{( \mu \mathbf { g } / \mathbf { g } \text { tissue) }}$ & & $0.27 \pm 0.08$ \\
Gr 1(Normal control) & $21.29 \pm 1.54$ & $3.84 \pm 0.38$ & $2.01 \pm 0.43$ & $0.24 \pm 0.05^{\#}$ \\
Gr II (MPTP control) & $6.53 \pm 0.86^{\#}$ & $1.37 \pm 0.21^{\#}$ & $0.38 \pm 0.21^{\#}$ & $0.26 \pm 0.08^{*}$ \\
Gr III (MPTP-MME) & $13.67 \pm 0.58^{*}$ & $2.63 \pm 0.19^{*}$ & $0.92 \pm 0.33^{*}$ & $0.24 \pm 0.08$ \\
Gr IV (MPTP-CPG) & $8.67 \pm 0.63$ & $1.41 \pm 0.15$ & $0.67 \pm 0.17$ & $0.24 \pm 0.08$ \\
Gr V (Curcumin Control) & $9.66 \pm 0.57$ & $1.83 \pm 0.22$ & $0.58 \pm 0.21$ & \\
\hline
\end{tabular}

of the protective effects curcumin have been linked with inhibition of inflammation coupled with anti-oxidation property during the acute phase of MPTP injury. Reactive oxygen species (ROS) formation is suggested to be involved in progressive dopaminergic neuron damage in PD.

\section{CONCLUSION}

In the present investigation, a mucoadhesive microemulsion system for intranasal delivery of Curcumin was successfully developed and optimized using BoxBehnken experimental design. The design is valid for predicting the globule size, PdI, viscosity, flux and \% drug release in the formulation optimization. From obtained results of above experiment, it can be concluded that 3\% Capmul MCM, 37\% of Accenon CC and Transcutol $\mathrm{HP}$ at 2.5:1 ratio, $0.5 \%$ sodium hyaluronate and $30 \mathrm{mg}$ of Curcumin, provide optimal globule size of $57.66 \mathrm{~nm}$ with narrow size distribution and suitable zeta potential of $-16.28 \mathrm{mV}$. In vivo studies indicate that mucoadhesive property of CMME comparatively helps to increase Curcumin uptake into the brain. Curcumin, through developed nasal mucoadhesive microemulsion system, increases DA concentration significantly in brain which in turn improves muscular coordination as well as gross behavioural activities of the test animal. Thus, intranasal formulation if properly formulated may be considered as an effective alternative approach to treat Parkinson's disease.

\section{ACKNOWLEDGEMENTS}

The authors would like to thank Arjuna Natural Extracts Pvt. Ltd., Hyderabad, India for providing Curcumin as gift sample. The authors thank Abitec Corporation, Jackson St Janesville, USA and Gattefosse, France for gifting oils, surfactants and co-surfactants. The authors are also thankful to Institute of Nuclear Medicine and Allied Sciences, Ministry of Defense, New Delhi, India for providing Male C57BL/6 mice. The authors are also grateful to Mrs. Kavitha Pillai for proof-reading the manuscript for grammatical and spelling errors.

\section{REFERENCES}

BARAKAT, N.S.; OMAR, S.A.; AHMED, A.A.E. Carbamazepine uptake into rat brain followingintraolfactory transport. J. Pharmacol. Pharmacother., v.58, n.1, p.63-72, 2006.

BARBIERO, J.K.; SANTIAGO, R.M.; LIMA, M.M.; ARIZA, D.; MORAIS, L.H.; VITAL, M.A. Acute but not chronic administration of pioglitazone promoted behavioral and neurochemical protective effects in the MPTP model of Parkinson's disease. Behav. Brain. Res., v.216, n.1, p.186$192,2011$.

BASSO, D.M.; BEATTIE, M.S.; BRESNAHAN, J.C. A sensitive and reliable locomotor rating scale for open field testing in rats. J. Neurotraum., v.12, n.1, p.21-26, 1995.

BROWN, S.M.; CAMPBELL, S.D.; CRAFFORD, A.; REGINA, K.J.; HOLTZMAN, M.J.; KHARASCH, E.D. $\mathrm{P}$-glycoprotein is a major determinant of norbuprenorphine brain exposure and antinociception. J. Pharmacol. Exp. Ther., v.343, n.1, p.53-61, 2012.

CHOPRA, S.; MOTWANI, S.K.; IQBAL, Z.; TALEGAONKAR, S.; AHMAD, F.J.; KHAR, R.K. Optimisation of polyherbal gels for vaginal drug delivery by Box-Behnken statistical design. Eur. J. Pharm. Biopharm., v.67, n.1, p.120-131, 2007.

DOUNA, H.; BAVELAAR, B.M.; PELLIKAAN, H.; OLIVIER, B.; PIETERS, T. Neuroprotection in Parkinson's disease: a systematic review of the preclinical data. Open. Pharmacol. J., v.6, n.1, p.12-26, 2012. 
ELSHAFEEY, A.H.; BENDAS, E.R.; MOHAMED, O.H. Intranasal microemulsion of sildenafil citrate: in vitro evaluation and in vivo pharmacokinetic study in rabbits. AAPS Pharm. Sci. Tech., v.10, n.2, p.361-367, 2009.

ESPOSITO, E.; DI MATTEO, V.; BENIGNO, A.; PIERUCCI, M.; CRESCIMANNO, G.; DI GIOVANNI, G. Nonsteroidal anti-inflammatory drugs in Parkinson's disease. Exp. Neurol., v.205, n.2, p.295-312, 2007.

GANNU, A.R.; RAO, Y.M. Formulation optimization and evaluation of microemulsion based transdermal therapeutic system for nitrendipine. J. Dispersion Sci. Technol., v.33, n.2, p.223-233, 2012.

GANNU, R.; PALEM, C.R.; YAMSANI, V.V.; YAMSANI, S.K.; YAMSANI, M.R. Enhanced bioavailability of lacidipine via microemulsion based transdermal gels: formulation optimization, ex vivo and in vivo characterization. Int. J. Pharm., v.388, n.1-2, p.231-241, 2010.

HARRIS, A.S.; SVENSSON, E.; WAGNER, Z.S.; LETHAGEN, S.; NILSSON, I.M. Effect of viscosity on particle size, deposition, and clearance of nasal delivery systems containing desmopressin. J. Pharm. Sci., v.77, n.5, p.405408, 1988 .

HIROMI, S.; KENJI, Y.; YASUSHI, S.; MANABU, F.; YUKIO, T.; KAZUTAKA, I.; RYOSUKE, T. A rotarod test for evaluation of motor skill learning. J. Neurosci. Meth., v.189, n.2, p.180-185, 2010.

HIRSCH, E.C.; HUNOT, S. Neuroinflammation in Parkinson's disease: a target for neuroprotection. Lancet. Neurol., v.8, n.4, p.382-397, 2009.

HOLCOMB, L.A.; DHANASEKARAN, M.; HITT, A.R.; YOUNG, K.A.; RIGGS, M.; MANYAM, B.V. Bacopamonniera extract reduces amyloid levels in PSAPP mice. J. Alzheim. Dis., v.9, n.3, p.243-251, 2006.

HORVAT, S.; FEHER, A.; WOLBUG, H.; SIPOS, P.; VESZELKA, S.; TOTH, A. Sodium hyaluronate as a mucoadhesive component in nasal formulation enhances delivery of molecules to brain tissue. Eur. J. Pharm. Biopharm., v.72, n.1, p.252-259, 2009.
LINDVALL, O.; EKDAHL, C.T.; CLAASEN, J.H.; BONDE, S.; KOKAIA, Z.; LINDVALL O. Inflammation is detrimental for neurogenesis in adult brain. Proc. Natl. Acad. Sci. USA, v.100, n.23, p.13632-13637, 2003.

LIU, G.P.; MA, Q.; SHI, N. Tyrosine hydroxylase as a target for deltamethrin in the nigrostriatal dopaminergic pathway. Biomed. Env. Sci., v.19, n.1, p.27-34, 2006.

MILLER, D.S.; BAUER, B.; HARTZ, A.M.S. Modulation of p-glycoprotein at the blood-brain barrier: opportunities to improve CNS pharmacotherapy. Pharmacol. Rev., v.60, n.2, p.196-209, 2008.

MANDAL, S.; MANDAL, S.D. Design and development of Carbamazepine mucoadhesive microemulsion for intranasal delivery: an ex-vivo study. Int. J. Pharm. Sci. Rev. Res., v.3, n.1, p.56-60, 2010.

MANDAL, S.D.; MANDAL, S.; PATEL, J. Development of curcumin loaded microemulsion drug delivery system for improving its dissolution profile. IJPFA., v.4, p.101-107, 2013.

MORIMOTO, K.; YAMAGUCHI, H.; IWAKURA, Y.; MORISAKA, K.; OHASHI, Y.; NAKAI Y. Effects of viscous hyaluronate-sodium solution on the nasal absorption of vasopressin and an analogue. Pharm. Res., v.8, n.4, p.471-479, 1991

PATEL, M.B.; MANDAL, S.; RAJESH, K.S. Formulation and kinetic modeling of curcumin loaded intranasal mucoadhesive microemulsion. J. Pharm. Bioallied. Sci., v.4, n.5, p.81-83, 2012.

PATHAK, R.; DASH, R.P.; MISRA, M.; NIVSARKAR, M. Role of mucoadhesive polymers in enhancing delivery of nimodipinemicroemulsion to brain via intranasal route. Acta. Pharm. Sin. B., v.4, n.2, p.151-160, 2014.

PIAOA, H.; BALAKRISHNANA, P.; CHOA, H.; KIMB, H.; KIMB, Y.; CHUNGA, S.; SHIMA, C.; KIM, D. Preparation and evaluation of fexofenadine microemulsions for intranasal delivery. Int. J. Pharm., v.395, n.1-2, p.309-316, 2010. 
REZNICHENKO, L.; KALFON, L.; AMIT, T.; YOUDIM, M.B.H.; MANDEL, S.A. Low dosage of rasagiline and epigallocatechin gallate synergistically restored the nigrostriatal axis in MPTP-induced parkinsonism. Neurodegener. Dis., v.7, n.4, p.219-231, 2010.
TOMOYUKI, F.; DAISUKE, I.; AKIKO, K.; YUTAKA, H.; TOSHIYASU, S. Influence of formulation viscosity on drug absorption following nasal application in rats. Drug. Metab. Pharmacokinet., v.22, n.3, p.206-211, 2007.

Received for publication on $09^{\text {th }}$ November 2015 Accepted for publication on $04^{\text {th }}$ January 2017 